\title{
The Lunar Environment: Determining the Health Effects of Exposure to Moon Dusts
}

Noreen Khan-Mayberry, Ph.D. ${ }^{\mp}$, NASA Johnson Space Center, 2101 NASA

Parkway, SF-23, Houston, TX 77058

\section{ABSTRACT}

The Earth's moon presents a hostile environment in which to live and work.

There is no atmosphere to protect its surface from the ravages of cosmic radiation, solar wind and micrometeorite impacts. As a result, the moon's surface is covered with a thin layer of fine, charged, reactive dust capable of entering habitats and vehicle compartments, where it can cause crewmember health problems. During the Apollo missions, lunar dusts were introduced into the Lunar Lander and command module, resulting in direct exposure and occasional reports of respiratory, dermal and ocular irritation. In order to further characterize the toxicological effects of lunar dust, NASA formed the Lunar Airborne Dust Toxicity Advisory Group (LADTAG). This interdisciplinary group is comprised of experts in space toxicology, lunar geology, space medicine, dust toxicity and biomedical research. Ultimately, this panel of experts will set health standards and risk criteria for use by vehicle design engineers, operations planners and astronauts during lunar missions.

¡ Author contact: noreen.n.khan-mayberry@nasa.gov; 281-483-1876 (office), 281-483-1876 (fax)

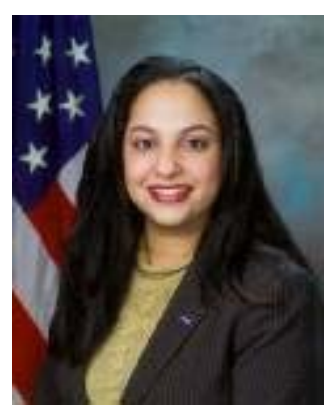




\section{INTRODUCTION}

The LADTAG group was formed as a direct result of a charge from NASA's Office of Chief Health and Medical officer (OCHMO). NASA's Chief Toxicologist and Life Sciences Pathologist were appointed to chair a committee of experts to include agency specialists and the world's leading experts on lunar dust, inhalation toxicology, lunar geology, space medicine and biomedical research. They selected this group of individuals from diverse technical backgrounds and also included an Apollo astronaut, to draw upon direct lunar surface experience. This group met and discussed the need for setting a lunar dust standard. Based upon LADTAG's qualified recommendations, it was determined to be prudent to pursue developing a permissible exposure limit (PEL) standard and human health risk criteria for lunar dusts.

Through targeted lunar research, LADTAG has already demonstrated that lunar soil contains several types of reactive dusts, including an extremely fine respirable component $(<10 \mu \mathrm{m})$, it has revealed that approximately $10 \%$ of lunar dust is in the respirable range, and that lunar dust has a very large surface area ( $\sim$ times that of a sphere of equivalent external size) [1]. These dusts have highly reactive surfaces in the lunar environment; the grains contain surface coatings which are generated by vapor phases formed by hypervelocity impact of micrometeorites. This unique class of dusts has surface properties that are unlike any earth based analog. These distinctive properties are why lunar dusts are of great toxicological interest. Understanding how these reactive components 
behave prior to being inhaled and then "biochemically" in a moisture-rich pulmonary environment will aid in determining how toxic these particles are to humans. The data obtained from toxicological examination of lunar dusts will determine the human risk criteria for lunar dust exposure and produce lunar dust standards.

This set of lunar dust standards, set to protect the health of our astronaut crews, will not only impact NASA medical operations, but will influence mission operation scenarios and engineering designs for vehicles and life support systems. The data from our multidisciplinary research are vital in developing dust remediation devices and lunar environmental monitors. Ultimately, the engineering and safety groups will design and develop countermeasures for space vehicles, suits, rovers and habitats that will be sustained within the limits of the health standard.

LADTAG's analysis of lunar dusts and lunar dust simulants will include detailed particle characterizations, determining the properties of particle activation, determining how to reactivate lunar dust, the process of dust passivation and discerning the pathological mechanisms of lunar dust exposure via inhalation, intratracheal instillation, cell culture exposure, dermal exposure and ocular exposure. The resulting set of health standards will be time-based and will vary by the duration and type of exposure. It may also be necessary to 
set multiple standards for different types of lunar dust, as well as for dust in its activated form vs. aged and passivated dust.

\section{EVOLUTION OF LUNAR SOILS}

Unlike terrestrial dusts, which are largely the product of atmospheric and hydrodynamic modification lunar dusts (the $<20 \mu \mathrm{m}$ fraction of lunar soils) undergo "space weathering", which is a generic term used for a number of processes that act on any body exposed to the harsh space environment [2]. The lunar surface does not have Earth's protective atmosphere, exposing it directly to repeated hypervelocity meteorite impacts (Figure 1). These meteorites are of a wide size range and velocity. Hypervelocity impact results in the communition or fragmentation of the lunar surface rocks and to a lesser extent fusion and vaporization. Communition gradually reduces the particle size of the lunar dust. Communition is balanced by agglutination which results when small volumes of soil are melted in a micrometeorite impact and the melt incorporates nearby mineral fragments to form a larger particle called an "agglutinate." .

Communition and agglutination are the two main dynamic processes involved in the textural evolution of lunar soils [3]. The texture of lunar soil evolves in a recurrent routine which is in direct response to continual reworking by the micrometeorite flux [4]. The reworking of the lunar soil by successive hypervelocity impacts is referred to as "gardening" the soil, this continuous process causes turn over, mixing and soil aggregation. During lunar operations we can expect communition to occur during module landing, when large amounts 
of regolith are displaced and crushed. Gardening can also occur as crews excavate soils for research and other operational use, such as radiation shielding.

Other space weathering processes on the lunar surface result from galactic and solar cosmic rays, irradiation, solar particle sputtering and implantation, and vapor deposition [3], [2]. Vapor and sputter deposits coat the majority of lunar soil grains in a mature soil, making these processes fairly important when considering the effects of space weathering [5] . Cosmic rays, which cause spallation (the process in which a bombarded nucleus breaks up into many particles), and radiation damage are considered less important to soil properties. Lunar geologists postulate that lunar grains, in the absence of an atmosphere and hydrosphere, are very reactive due to their grain surfaces being etched by solar wind; such surfaces have a high density of crystal dislocations, see Figure 2, [6]. The weight of evidence from lunar geology research, spanning the past $10-20$ years, has demonstrated that that virtually all available lunar dust samples contain vapor deposited nanophase iron $\left(\mathrm{np}-\mathrm{Fe}^{0}\right)$ embedded in impact glass; see Figure 3 [7], [8]. It is key to note that there is no known surface passivation mechanism for lunar particles other than agglutination [1].

\section{LUNAR CONCEPT OF OPERATIONS}

When determining a plan of action for lunar dust toxicity research, LADTAG considered the health of the astronauts during various plans of anticipated lunar operations. The lunar concept of operations influences certain 
aspects of how research is conducted. Specific questions that LADTAG research attempts to answer are based upon the needs outlined in the concept of operations, such as the need to develop a short term standard for lunar contingency operations. NASA has been presented some assumptions regarding the lunar concept of operations plan. This plan is based upon input from NASA experts in mission operations, mechanical systems, life support, and space life sciences.

The current concept plans for landing on the moon is to pre deploy the Lander and Earth Departure Stage (EDS) to low Earth orbit (See Figures 4 \& 5). The Lander is a two stage, expendable module which consists of a crew cabin and an integral airlock, probably separate. The Orion vehicle will then be launched with the crew and will Rendezvous with the Lander and EDS. Orion consists of a command module (CM), service module (SM) and launch abort system (LAS). Once docked, the CEV-Lander will travel to Lunar orbit; at that point the crew members will transfer to the Lander, undock from Orion, and descend to the lunar surface. Orion is left unoccupied in low lunar orbit. After the lunar stay, the ascent stage of the Lander will Rendezvous with Orion, returning the crew and cargo (lunar samples.) Orion will undock from the Lander and return the crew to Earth's atmosphere by skip or direct entry. The Lander is disposed via impact on the lunar surface. Lunar surface activities will introduce dust into the habitat and Lander. Rendezvous of the Lander with Orion provides another dust pathway into that module, too. 
The concept plan gives us other details to consider for long duration stays, such as the storage site of the lunar rover, the type of storage facility, design of rover (i.e., is the rover sealed?). All these factors will affect astronaut exposure times. We expect a substantial amount of extravehicular activity being performed during each mission. The crew members will be working with lunar materials on the surface for various reasons including performing experiments. The use of plant material may become an integral part of the lunar habitat and the potential introduction of possible toxic byproducts must be contemplated. The management of wastes must be carefully planned and should not consist of introduction of pyrolysis products in the lunar habitat.

\section{THE APOLLO EXPERIENCE}

This is not NASA's first visit to the moon, so the Apollo missions serve as a vital resource for planning our return to the lunar surface. The Apollo experience has told us that dust could be a dermal, ocular and respiratory irritant [9]. There are several accounts from Apollo crewmembers. In crew debriefs, some referred to lunar dust as being similar to sandpaper, having a "texture like graphite", stating that the dust "immediately got into" their eyes, giving a "distinct gunpowder like scent", having a "cloud of fine dust floating around the lunar module", "throat irritation", nasal passage irritancy, "tasting" and "eating" lunar dust particles, skin irritation from lunar dust entering gloves and spacesuits. The mission reports and accounts of the crews give us some measure of brief, episodic exposure and insight into some expected effects. 
A review of the Apollo crews' health effects post lunar exposure do not necessarily negate the toxicity concerns of lunar dust. While the Apollo experience is invaluable, there are several limitations that we must consider. The exposure data from Apollo are considered a reference due to a number of factors. First, the Apollo crewmember exposures to lunar dust occurred once the crew returned to zero gravity, at this point the dust had been aged in the Lunar Lander for several hours. The exposures were also uncontrolled, not measurable, brief and episodic. The crews were also too small of a group (low $n$ ) to be considered test subjects. Further distinctions include Apollo mission operations duration of exposure and the concentrations of lunar dust. Exploration missions, even the early lunar sortie missions, are expected to have a greater number of extravehicular activity (EVA), thereby increasing exposure duration and airborne concentrations. The potential for exposure to lunar dust will be immediate, resulting in exposure to "fresh" or "active" (non-aged) lunar dust, as the crews' conduct multiple EVA's and traverse in and out of the LSAM. The long term operation differences also include building a lunar habitat and long duration occupation of the lunar surface.

In order to get a glimpse into the types of dusts that were introduced to the Apollo vehicle and the astronauts directly, LADTAG is conducting research in forensic engineering. The forensic engineering group consists of lunar geologists that are examining Apollo artifacts including space suits (See Figure 6), air filters, 
$\mathrm{LiOH}$ canisters, which are $\mathrm{CO}_{2}$ absorbing devices (See Figure 7), sample collections boxes, vacuum bags (bags from vacuum cleaners used to remove dirt and dust) and washings (dust particle residue isolated from Freon washing of samples boxes). This work also provides key information about the size range of particles.

\section{TOXICITY OF DUSTS}

There is quite a bit of data on the toxicity of terrestrial dusts. This bank of terrestrial data provides a reference when determining the toxicity of lunar dusts. The closest Earth based analogs to lunar dusts are mining-generated dusts and volcanic ash. Volcanic ash analogs are useful for creating lunar simulants. They also provide evidence of toxicity in humans that can be use as a comparable model.

In regards to lunar dusts there are several health questions that we have yet to answer. Several attributes of lunar dust cause concern when considering possible health effects to respiratory, dermal, ocular and cellular systems. Lunar dusts are highly abrasive, which could potentially erode the dermal water vapor barrier (the outer skin layers that are in place to keep internal water and heat from escaping the human body). Ocular concerns include effects ranging from minor irritation, physical or chemical injury. Respiratory injury could include compromised lung clearance mechanisms and the effectiveness of lung injury in a 1/6 g environment. Some fundamental questions include; how will the lower 
respiratory system handle the highly unusual shapes of lunar dust particles? Does cellular activity affect lung toxicity? Cellular level concerns include the generation of reactive oxygen species (ROS) in lung epithelia and lung macrophages. More questions to answer include dust reactivity. How quickly will these particles passivate? Does passivation decrease lunar particle toxicity? What is the effect of $n p-\mathrm{Fe}^{0}$ on toxicity?

\section{LADTAG's PLAN OF ACTION}

LADTAG began making decisions on necessary research efforts in early 2006. LADTAG couples guided research efforts and standard setting. This research is necessary to fill data gaps. LADTAG's external members serve as non-advocate reviewers of directed research. LADTAG will ultimately integrate all data obtained from each directed research arm (DRA) of the LADTAG project. The directed research arms consist of Toxicology (respiratory), Chemistry (cell culture, chemical characterization and heat activation), Ames Biology (cell culture, ROS generation in lung, dermal toxicity, and ocular toxicity) and Lunar Geology (characterization, size distribution, solar wind implantation \& activation, forensic engineering, clam shell and skim scoop samples). All directed research principal investigators will first test their assays on lunar simulant, since actual lunar dust is limited in quantities. After proving their methods to be effective, each group will then test highland mature and highland immature lunar dusts. These dusts were selected since they most closely resemble what our crews will be exposed to at the South polar landing site. 
LADTAG has determined several research gaps for each DRA. The goal of our studies is to fill these research gaps. Closing these gaps will determine the factors that will be used to calculate the lunar dust standard. The factors include species extrapolation, adjustment for exposure times, surface activation and size distribution factors.

The Lunar Geology DRA has been tasked to complete several projects. This group consists of several teams that will conduct research to fill data gaps and provide technical oversight for several lunar geology projects. One team will characterize lunar dust recovered from Apollo hardware (forensic engineering). Another team is charged with the characterization and size distribution of lunar dust from pristine, unfractionated Apollo soils, the determination of an accurate grain size distribution for major representative lunar soil types and the mineral and glass phase identification and populations for major representative lunar soil types. Yet another team will activate simulants with hydrogen/proton bombardment and UV in order to compare surface reactivity with non-activated soils. Clam shell (See Figure 8) and skim scoop samples will be studied by another team. These clam shell samples are from the uppermost layer of the lunar surface and will provide insight into the dust size distribution that crew members are most likely to be exposed to. Ultimately Lunar Geology's DRA will provide new data on the finest fraction ( $<1$ micrometer) and will characterize soil from Apollo hardware, from pristine lunar soil samples, and from activated 
samples. The Lunar Geology DRA will deliver lunar dust to toxicology team for research testing.

The Chemistry DRA will work jointly with the Lunar Geology DRA to perform lunar dust dissolution studies. This joint effort will determine the rate of lunar dust dissolution in neutral $\mathrm{pH}$ and in reduced $\mathrm{pH}$, characteristic of lysosomal $\mathrm{pH}$. The Chemistry DRA is also working to determine the properties of lunar activation using various techniques including heat activation, uncovering the rate of dust passivation and exposing the lunar dust to simulated solar wind bombardment. Finally, the Chemistry DRA is working jointly with the Ames Biology DRA in the investigation of reactive oxygen species (ROS) generation in lung tissue culture; this work will compliment the work of the respiratory studies.

The Ames Biology DRA will also evaluate the effects of lunar dust on the skin. The Ames Biology DRA will determine the potential for lunar dust to cause skin irritation and skin sensitization. Experimental systems will be used, including in vitro skin models and animal subjects. Skin studies will also include abrasion studies, in order to help define permissible limits for skin entry into the space suit. The Ames Biology DRA is also responsible for conducting studies of the ocular effects of lunar dust. These studies will involve both in vitro and in vivo experimental systems, in order to assess the potential for lunar dust to damage the eyes, and to provide a means for evaluating the effectiveness of treatment methods for dealing with dust entry into the eyes. 
Finally, the Toxicology DRA will focus on conducting intratracheal instillation (ITI) and inhalation studies of simulants and lunar dusts. The Toxicology DRA will determine the direct toxicity of lunar dust in the respiratory system, the rate of deposition, and the effects of active and passivated dusts. This work is critical in setting standards for lunar dust. The data obtained from the Toxicology DRA will establish the species extrapolation factor and contribute to the surface activation factor. Both of these factors along with the time and distribution factors will be used to calculate the lunar dust standard.

\section{THE ULTIMATE DELIVERABLE: RISK CRITERIA \& HEALTH STANDARDS}

As all data are completed and integrated LADTAG will set defensible exposure limits for lunar dusts. LADTAG will set risk criteria based upon research findings that have filled our stated research gaps. Ultimately, LADTAG will publish research findings in peer-reviewed publications and interface with other NASA groups that are studying lunar dusts. Development of time-based standards, acute exposure limits, exposures of a few hours, and chronic exposure limits, episodic exposures up to six months, for inhalation (pulmonary) toxicity and human risk criteria will be developed no later than 2010. LADTAG does not rule out the development of setting other (non pulmonary) standards and human health risk criteria, for dermal and ocular exposure, contingent upon research findings of non-airborne dust toxicity studies [9]. 


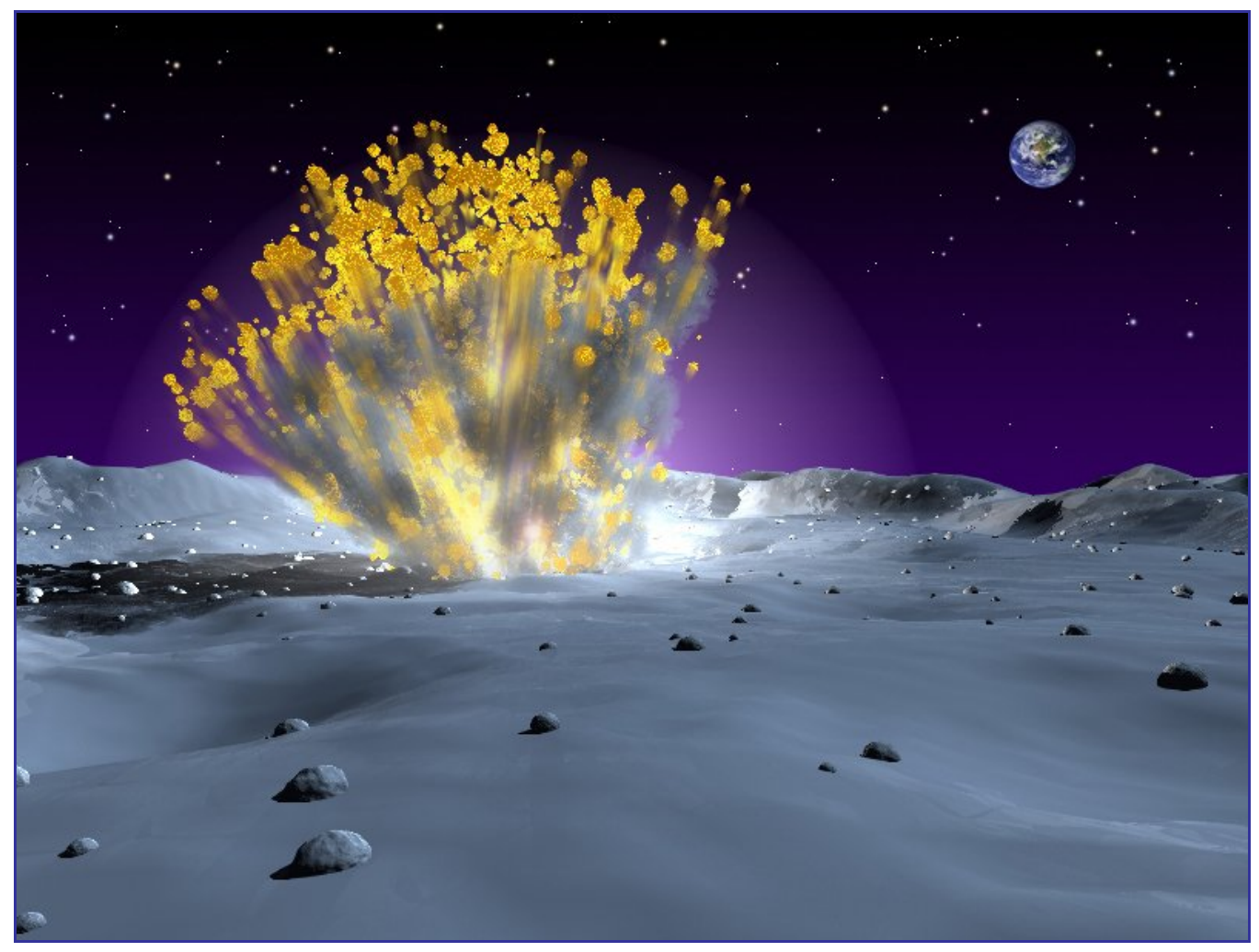

Figure 1. Artist's rendition of meteorite bombardment on lunar surface.

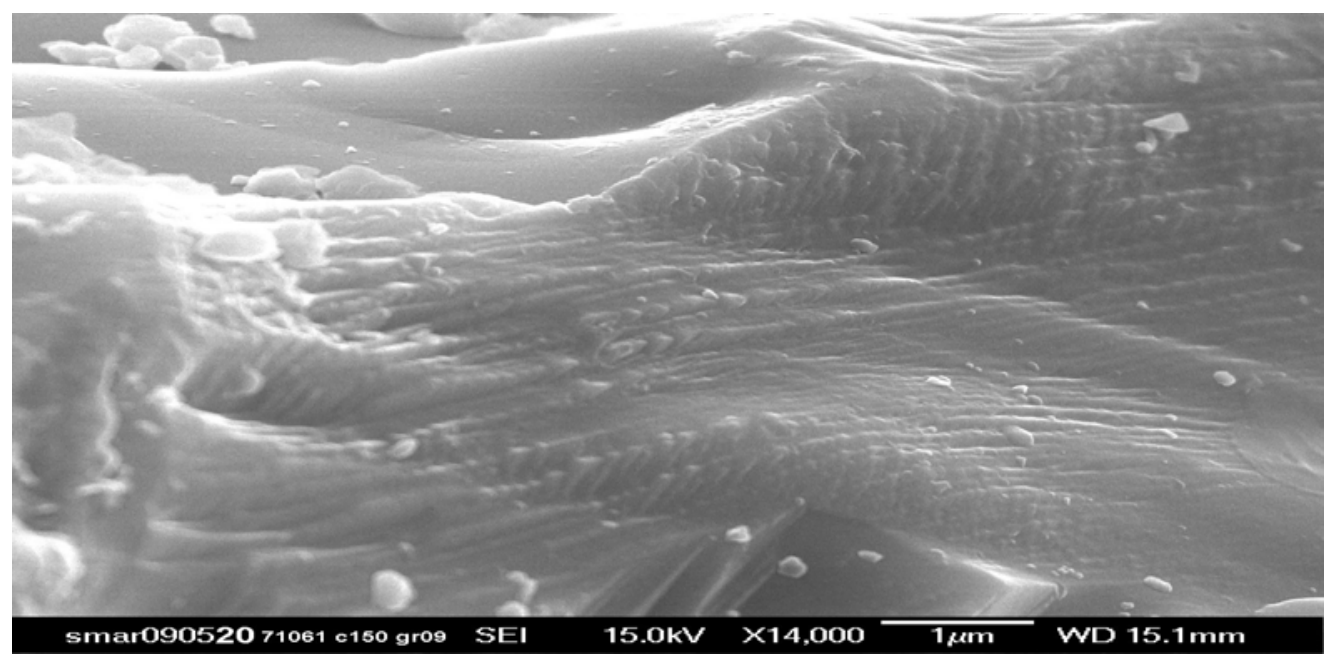

Figure 2. Lunar Grain Surface Etched by Solar wind. 


\section{Synthesis of Lunar Glass Simulant With NanoPhase Metallic Fe}

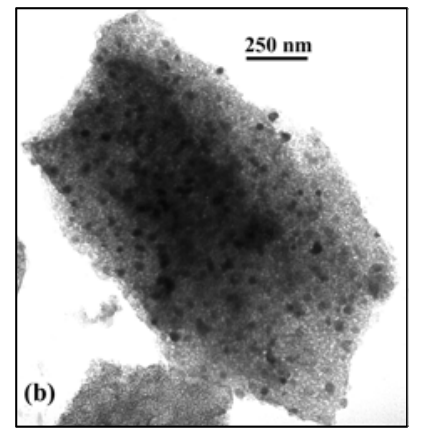

\section{TEM Photos}
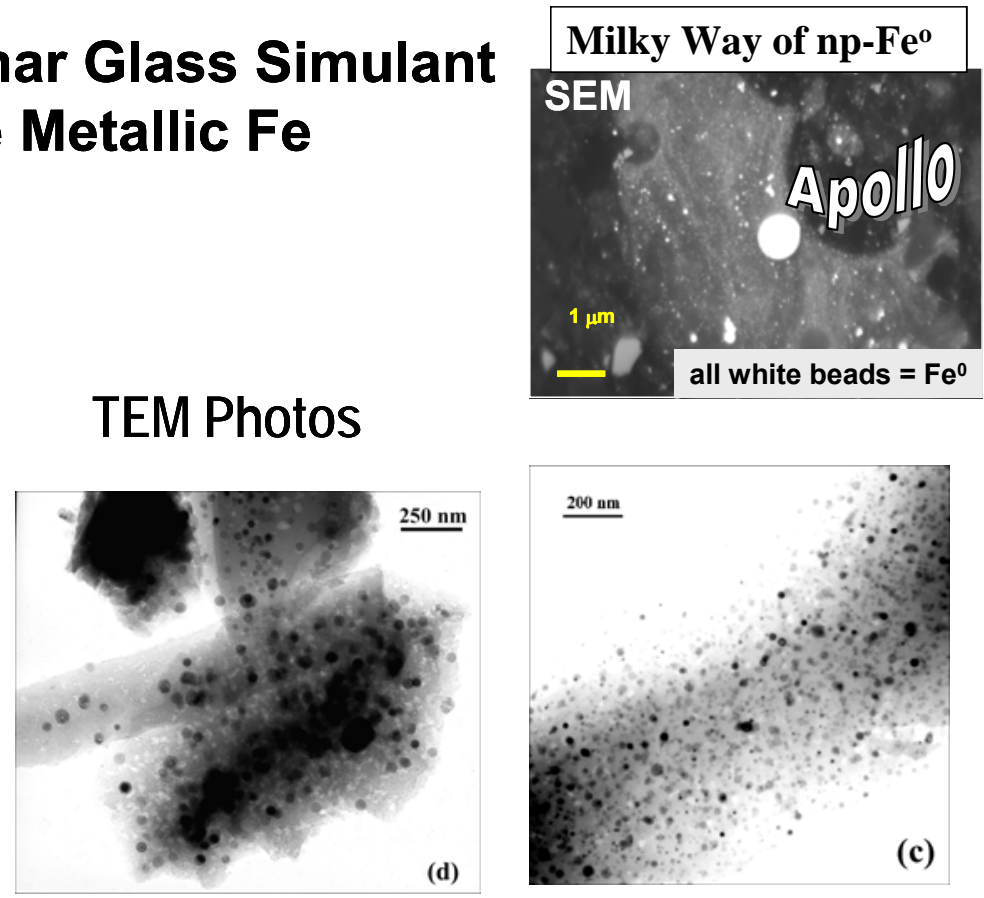

All Black Dots are Nanophase Fe

(d)

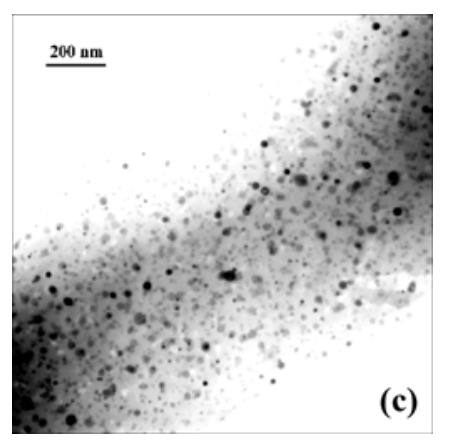

Figure 3. TEM photos of synthetic nanophase Fe suspended in silica-rich glass. With TEM, all black spots are nanophase Iron (np-Fe ${ }^{0}$. 


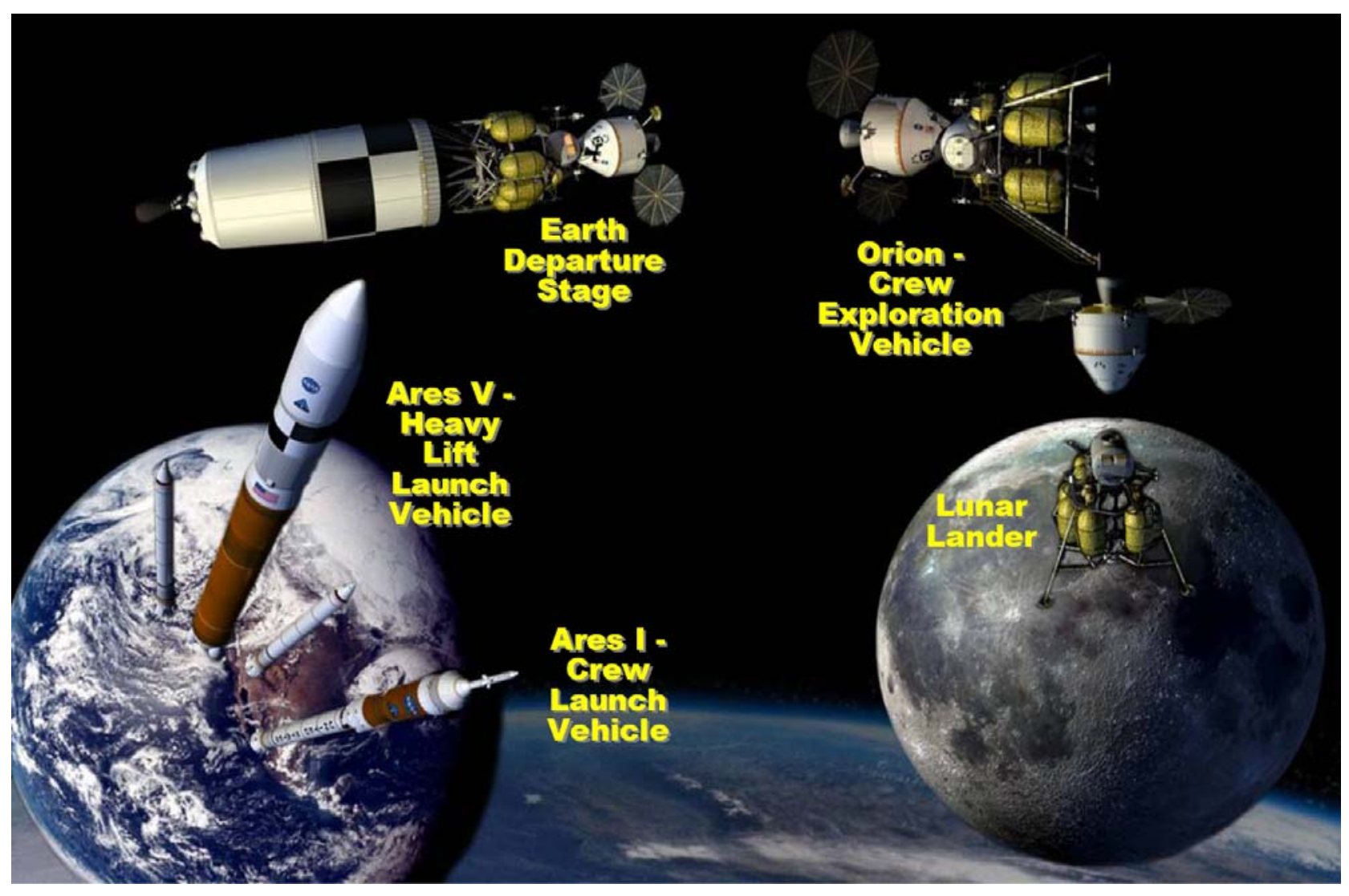

Figure 4. Components of Program Constellation 


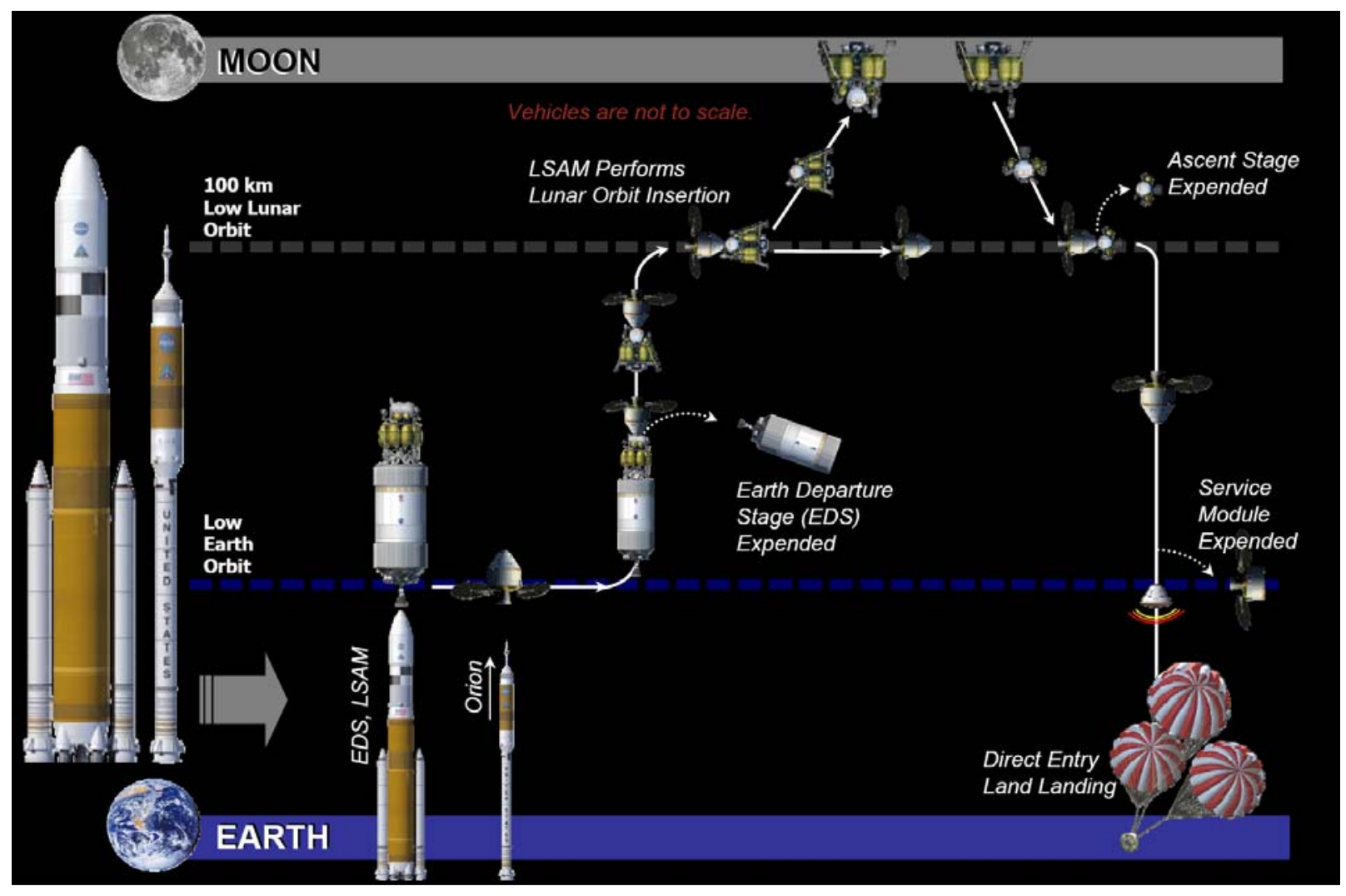

Figure 5. Typical Lunar Reference Mission 

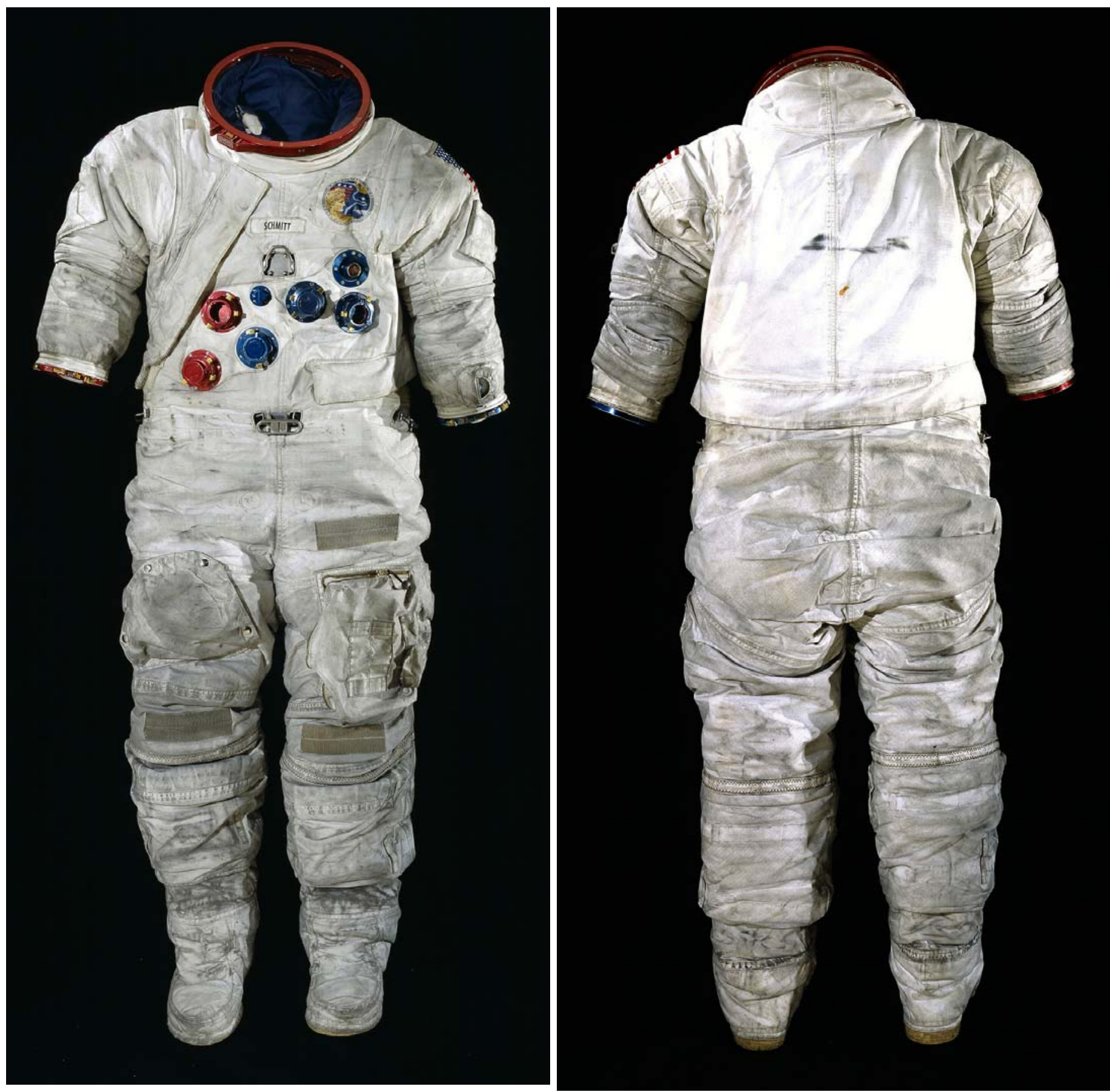

Figure 6. Apollo Astronaut Harrison "Jack" Schmitt's Suit (front \& rear view) covered with lunar dust 


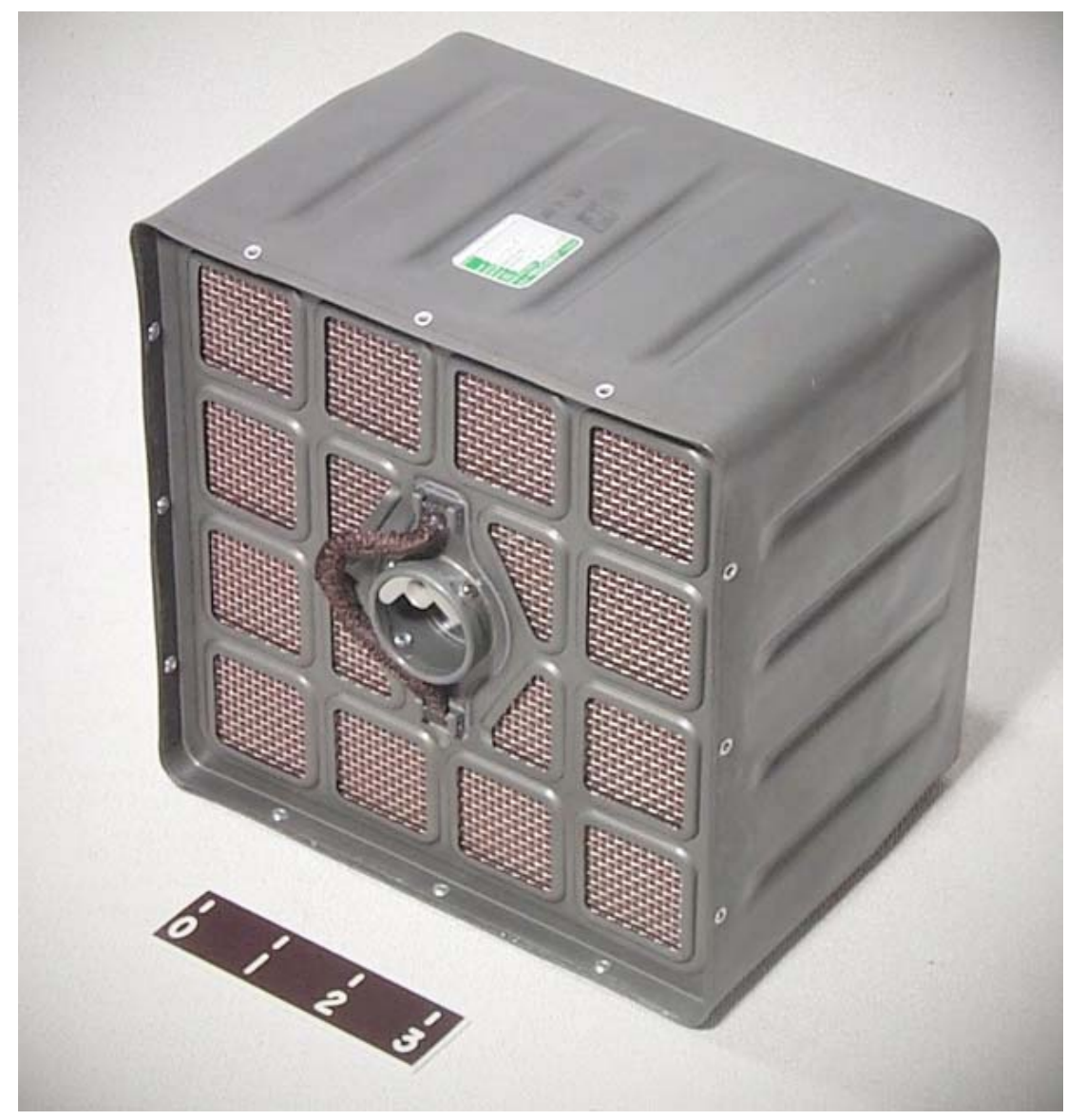

Figure 7. Apollo $\mathrm{LiOH}$ canister ( 2 canisters used in parallel, changed out every 24 hours during mission) Top - LiOH filter inlet. Bottom - Artist's rendition.

$\mathrm{CO}_{2}$ ABSORBER FILTER ASSEMBLY

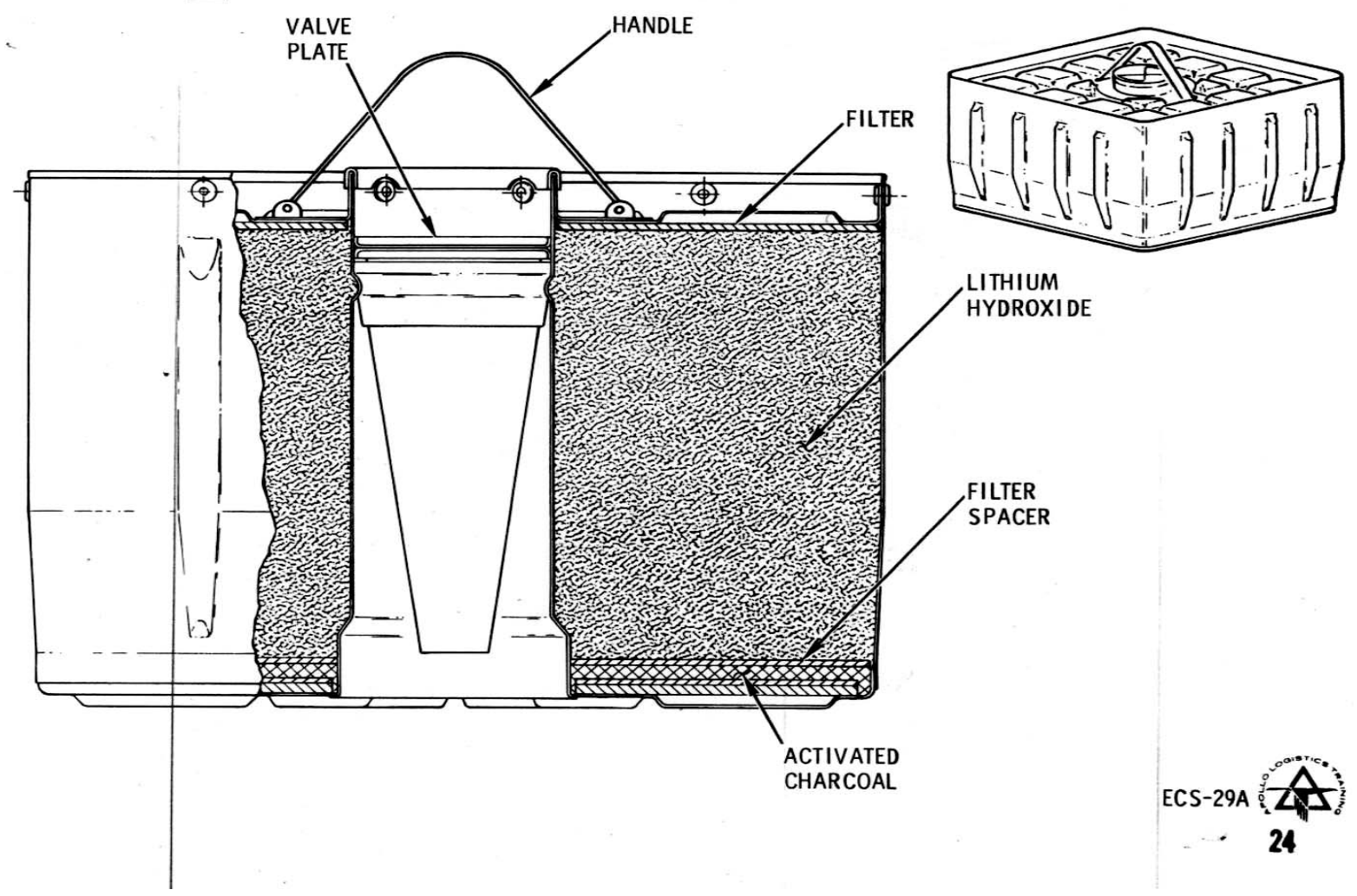




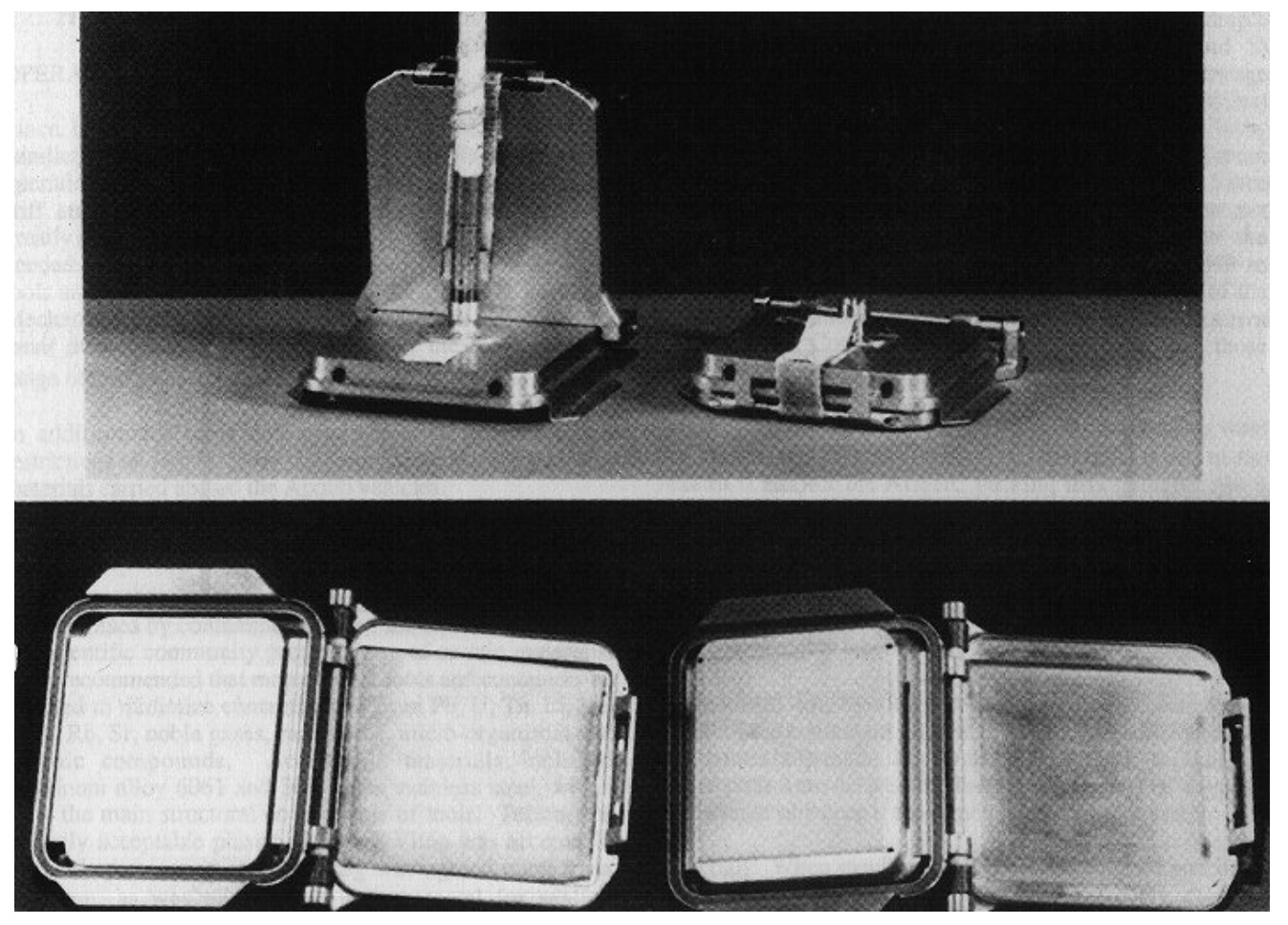

Figure 8. Clam Shell Sampling Device (upper) and clam shell sample with dust (lower). 


\section{REFERENCES}

[1] James, J.T. (2007) Lunar Dust Inhalation Standard. Exploration Science Mission Directorate meeting. February 1, 2007, New Orleans, Louisiana. NASA Johnson Space Center, MS-SF2, Houston, Texas.

[2] Noble, S.K. and Lindsay, J. (2007) The Microscopic Moon: Space Weathering of Lunar Soil. Presented at 2007 NESC Lunar Dust Workshop, January 30February 2, 2007, Ames Research Center, Moffett Field, California. NASA Johnson Space Center, MS-KA, Houston, Texas.

[3] Keller L. P and McKay D. S. (1997) The nature and origin of rims on lunar soil grains. Geochimica et Cosmochimica Acta 61:2331-2341.

[4] McKay, D.S. et al., 1991, The lunar regolith. In The Lunar Sourcebook: A User's Guide to the Moon. Cambridge Univ. Press, 285-356.

[5] Keller L. P., Wentworth S. J., McKay D. S., Taylor L. A., Pieters C. M. and Morris R. V (2000) Space weathering in the fine size fractions of lunar soil: Mare/highland differences LPSCXXXI, ab\#1655). Lunar and Planetary Institute, Houston TX (CDROM). 
[6] McKay, D.S. (2006) Lunar Geology Overview. Presented at Lunar Airborne Dust Advisory Toxicity Group, $3^{\text {rd }}$ Meeting, December 7-8, 2006, Houston, Texas. NASA Johnson Space Center, MS-KA, Houston, Texas.

[7]Taylor, L.A. (2006) Lunar Geology Characterization. Presented at Lunar Airborne Dust Advisory Toxicity Group, $3^{\text {rd }}$ Meeting, December 7-8, 2006, Houston, Texas. Planetary Geosciences Institute - University of Tennessee Knoxville.

[8] Liu, Y., Taylor, L.A., Thompson, J.R., and Parl, J., 2006c, Unique properties of lunar impact glass: Simulating nanophase iron in agglutinitic glass. Amer. Mineral., in press.

[9] Khan-Mayberry, N.K. (2007) Lunar Airborne Dust Toxicity Advisory Group. Presented at 2007 NESC Lunar Dust Workshop, January 30-February 2, 2007, Ames Research Center, Moffett Field, California. NASA Johnson Space Center, MS-KA, Houston, Texas.

Acknowledgements: I would like to acknowledge the following persons for their work towards setting lunar dust standards: Dr. John T. James, Dr. Russell L. Kerschmann, Dr. Chiu-wing Lam, Dr. Antony Jeevarajan, Dr. David J. Loftus, Dr. David S. McKay, Dr. Lawrence Taylor, Dr. John Lindsay, Dr. Sarah K. Noble and Dr. Thomas A. Sullivan. 